\title{
An Improved Soft Decision Based MIMO Detection Using Lattice Reduction
}

\author{
Mehnaz Rahman, Ehsan Rohani, Jingwei Xu, and Gwan S. Choi
}

\begin{abstract}
This paper presents a performance analysis of soft decision based Lattice Reduction (LR) aided Schnorr-Euchner (SE) multiple-input-multiple-output (MIMO) detection algorithm with that of a hard one. We develop an advanced soft decoding scheme, for $4 \times 4$ MIMO with different modulation schemes and it provides 1.3 to $1.5 \mathrm{~dB}$ improvement compared to the LR-aided hard decision based detection method. The optimum parameters for $K$ and saturation limit are further derived using extensive simulation. In the conventional K-best algorithm limiting the LLR values is not beneficiary in reducing the optimum size of candidate list, whereas applying the saturation limit on the LLR values in LR-aided algorithm will result in more than $8 x$ reduction in list size as well as in the complexity of detector and LLR calculation unit.
\end{abstract}

Index Terms-MIMO, soft decoding, lattice reduction, and k-best detector.

\section{INTRODUCTION}

MIMO communication systems have been adopted by advanced wireless standards such as IEEE 802.11n, IEEE 802.16e and 3GPP Long Term Evolution (LTE) to achieve increased spectral efficiency and high throughput data rates. Most of these standards have a specified minimum error rate to guarantee Quality of Service (QoS), which presents as an exact value in bit error rate (BER) or packet error rate (PER). As an example, $10^{\wedge}-6$ is specified as the minimum required BER in IEEE 802.16e standard [1].

The main challenge in the use of MIMO systems is the computation power and complexity necessary for detection of received signal at the receiver side. Several algorithms have been proposed to address this complexity, offering different tradeoffs between power and performance. The maximum likelihood (ML) detector minimizes BER performance through exhaustive search, but complexity grows exponentially with increasing number of antennas. In contrast, linear detectors (LDs) and successive interference cancellation (SIC) detectors requiring polynomial complexity suffer from significant performance loss. Recently, lattice reduction (LR)-aided detection has been proposed in [2]-[4] which show that LR-aided detectors can achieve the same diversity as the ML detector but exhibit some performance loss compared to ML detectors [5]. Suboptimal detectors can be extended further to provide soft-output detectors and soft input-soft output (SISO) detectors that are suitable for subsequent iterative decoding

Manuscript received November 11, 2013; revised April 2, 2014.

The authors are with the Department of Electrical and Computer Science, Texas A\&M University, College Station, TX 77840, USA (e-mail: mehnaz@tamu.edu,_ehsanrohani@tamu.edu,_xujw@tamu.edu, gchoi@ece.tamu.edu).
[6], [7].

The LR-aided K-best tree search algorithm was first proposed in [8] and [9] and improved in [10]. Compared to a conventional K-Best search, the LR-aided K-best algorithm has no boundary information about the symbols in the lattice-reduced domain. Practically, this means that the set of children in a given layer is infinite, as it is unconstrained. This infinite set is replaced by a finite subset in [8] and [9] developing a so-called "on demand" child expansion based on the Schnorr-Euchner (SE) strategy [10] for reducing the complexity of generating the subset. The algorithm is further improved by the addition of a priority queue and on demand child expansion in [11]. Then it is extended to the complex domain in [12].

In this paper, we develop a soft decision based LR-aided K-best decoder, which enables the utility of lattice reduction in soft decision as well as reducing the gap between the $\mathrm{K}$-best decoder and the maximum likelihood optimal decoder. In order to perform the soft decoding, we first compute the LLR values from the $\mathrm{K}$ best candidates at the last layer of conventional LR-aided MMSE extended K-best search. Then the LLR values are fed into an LDPC decoder and the results have been compared with the result of hard decision made on the best candidate of the last layer. As the candidate list is a subset of the tree, further optimization on list size is proved to be possible with limiting LLR values. A saturation of plus and minus eight is applied to limit the over confident LLRs that are the result of lack of hypothesis in candidate list. Combining LR, MMSE extension, and LLR clipping we were able to reduce the minimum required list size and also improved performance (e.g. for $4 \times 4$ antenna arrangement and 16 QAM compared with conventional K-best the list size will reduce from 256 [13] to 64 and compared to improved system without LLR clipping the list size is reduced more than eight times).

The rest of this paper is organized as follow. In Section II we introduce the MIMO detection problem based on LR-aided MMSE extended tree search algorithm. In addition, we have discussed the soft decoding of the algorithm in that section. Later in Section III we have analyzed the results for all of our studied cases and finally Section IV concludes this paper with a brief overview.

\section{SYSTEM MODEL}

The transmission model of a MIMO system with $N_{T}$ transmit antenna and $N_{R}$ receiving antenna and operating in symmetric M QAM scheme is:

$$
y^{c}=H^{c} s^{c}+n^{c}
$$


where $s^{c}=\left[s_{1}, s_{2}, \ldots . s_{N_{T}}\right]^{T}$ is the $N_{T}$ dimensional complex information symbol vector transmitted, $H^{c}$ is complex channel matrix and $y^{c}=\left[y_{1}, y_{2}, \ldots y_{N_{R}}\right]^{T}$ is the $N_{R}$ dimensional complex information symbol vector received Noise vector, $n^{c}=\left[n_{1}, n_{2}, \ldots . n_{N_{R}}\right]^{T}$ is a $N_{R}$ dimensional circularly symmetric complex zero-mean Gaussian noise vector with variance $\sigma^{2}$. The corresponding real signal mode is:

$$
\begin{gathered}
{\left[\begin{array}{l}
\mathfrak{R}\left[y^{c}\right] \\
\mathfrak{I}\left[y^{c}\right]
\end{array}\right]=\left[\begin{array}{cc}
\mathfrak{R}\left[H^{c}\right] & -\mathfrak{I}\left[H^{c}\right] \\
\mathfrak{I}\left[H^{c}\right] & \Re\left[H^{c}\right]
\end{array}\right]\left[\begin{array}{l}
\mathfrak{R}\left[s^{c}\right] \\
\mathfrak{I}\left[s^{c}\right]
\end{array}\right]+\left[\begin{array}{l}
\mathfrak{R}\left[n^{c}\right] \\
\mathfrak{I}\left[n^{c}\right]
\end{array}\right]} \\
y=H s+n
\end{gathered}
$$

where $s=\left[s_{1}, s_{2}, \ldots . s_{2 N_{T}}\right]^{T}, y=\left[y_{1}, y_{2}, \ldots . y_{2 N_{R}}\right]^{T}$ and . $n=\left[n_{1}, n_{2}, \ldots . n_{2 N_{R}}\right]^{T}$. The real and imaginary parts of a complex number are denoted by $\Re(\cdot)$ and $\mathfrak{J}(\cdot)$ respectively. ML detector solves for the transmitted signal by calculating:

$$
\hat{s}=\arg _{\bar{s}_{\in} S^{2 N_{T}}} \min \|y-H \tilde{s}\|^{2}
$$

Here $\|\cdot\|$ denotes the 2-norm. This MIMO detection problem is also known as closest point problem in [14] that represents a search through the set of all possible lattice points. Each antenna provides a 2-level search: one for detecting real part and the other for imaginary part of transmitted symbol of that antenna. The distance between a received signal and a proposed hypothesis in $2 N_{T}$ dimensional space is called the Euclidean distance which measures the error of that hypothesis. The search is satisfied by the solutions with minimum error. LR-aided detection is used to reduce the complexity of the ML detector [8]. Since lattice reduction requires unconstrained boundary, so the following change is made to (3) to obtain a relaxed search:

$$
\hat{s}=\arg _{\bar{s} \in \mathcal{U}^{2 N_{T}}} \min \|y-H \tilde{s}\|^{2}
$$

where $U$ is unconstrained constellation set as $\{\ldots,-3,-1,1,3, \ldots\}$. But $\hat{s}$ may not be a valid constellation point, so a quantization step is applied:

$$
\hat{S}^{N L D}=\mathcal{Q}(\hat{s})
$$

where $Q($.$) is the symbol wise quantizer to the constellation$ set $S$. It is equivalent to naive lattice detection (NLD) studied in [15] and [16]. But the proposed NLD does not generally have good diversity-multiplexing tradeoff (DMT) optimally, even with the $\mathrm{K}$ best search [11]. To achieve DMT, the following modifications are proposed in [15] and [11]:

$$
\begin{gathered}
\hat{s}=\arg _{\bar{s} \in \mathcal{U}^{2 N_{T}}} \min \left(\|y-H \tilde{s}\|+\frac{N_{0}}{2 \sigma_{s}^{2}}\|\tilde{s}\|\right) \\
\hat{s}=\arg _{\bar{s} \in \mathcal{U}^{2 N_{T}}} \min \|\bar{y}-\bar{H} \tilde{s}\|^{2}
\end{gathered}
$$

Here we have included MMSE regularization, $E\left\{s S^{T}\right\}=$ $\sigma_{S}^{2} I$ with $I$ as a $N \times N$ identity matrix, and $\bar{H}$ and $\bar{y}$ in (6) are the MMSE extended channel matrix and received signal vector defined as:

$$
\bar{H}=\left[\begin{array}{c}
H \\
\sqrt{\frac{N_{0}}{2 \sigma_{2}^{2}}} I_{2 N_{T}}
\end{array}\right], \bar{y}=\left[\begin{array}{c}
y \\
0_{2 N_{T} \times 1}
\end{array}\right]
$$

where $0_{2 N_{T} \times 1}$ is a $2 N_{T} \times 1$ zero matrix. LR- aided detectors apply lattice reduction to the matrix $\bar{H}$ to find a more orthogonal matrix $\widetilde{H}=\bar{H} T$, where $T$ is a unimodular matrix. This reduction effectively finds a better basis for the lattice defined by the channel matrix, thereby reducing the effect of noise and minimizing error propagation. After the reduction, the NLD with MMSE becomes

$$
\hat{s}=2 T \arg \min _{\tilde{z} \in \mathbb{C}^{2 N_{T}}}\left(\|\tilde{y}-\widetilde{H} \tilde{z}\|^{2}+1_{2 N_{T} \times 1}\right)
$$

where $\tilde{y}$ is the complex received signal vector and $1_{2 N_{T} \times 1}$ is a $2 N_{T} \times 1$ one matrix. After shifting and scaling of (8), we obtain:

$$
\hat{s}=2 T \tilde{z}+1_{2 N_{T} \times 1}
$$

\section{A. K-Best Search}

The K-Best search with lattice reduction proposed in [8] and [9] belongs to a particular subset of the family of breadth-first tree search algorithms. At a high algorithmic level of abstraction, the LR aided $\mathrm{K}$ best search is performed sequentially, solving for the symbol at each antenna. At first, it does $\mathrm{QR}$ decomposition on $\widetilde{H}=Q R$, where $\mathrm{Q}$ is a $2\left(N_{R}+N_{T}\right) \times 2 N_{T}$ orthonormal matrix and $\mathrm{R}$ is a $2 N_{T} \times 2 N_{T}$ upper triangular matrix. Then (8) is reformulated as

$$
\hat{s}=2 T \arg \min _{\tilde{z} \in \mathbb{Z}^{2 N_{T}}}\left(\|\breve{y}-R \tilde{z}\|^{2}+1_{2 N_{T} \times 1}\right)
$$

where $\breve{y}=Q^{T} \tilde{y}$. The error at each step is measured by the partial Euclidean distance (PED), e.g. the accrued error at a given level of the tree, for a given path through the tree.

For an arbitrary level of the tree, the $\mathrm{K}$ best nodes are collected, and passed to the next level for consideration. At the end, the K paths through the tree are evaluated. While working with hard decision, the path with the minimum overall error is selected as the most likely solution. In contrast for soft decision, each path of chosen $\mathrm{K}$ best paths is considered as potential candidates. Therefore, all of the chosen paths are passed to the LLR update unit for LLR calculation. The LLR values are then fed into the LDPC decoder for the final decoding.

\section{B. On Demand Expansion}

The principle of the on demand expansion scheme is that children of a given node in the tree can be enumerated in a strictly non-decreasing error order. It employs the Schnorr-Euchner (SE) strategy to perform an on-demand child expansion, where a child is expanded if only if all its better siblings are expanded and chosen as the partial candidates of the $n^{\text {th }}$ layer. A significant reduction on the node expansions is achieved in [7], but both algorithms in [6]-[7] require at least $O\left(N_{T} K+K^{2}\right)$ operations to find the exact $\mathrm{K}$ best partial candidates for each layer. We have used algorithm in [7] to perform the list calculation. When working with hard decision, we compute the final solution by 
choosing the path with the minimum overall error. But for soft decision, each path of chosen $\mathrm{K}$ best paths is considered as potential candidates and passed to the soft input soft output decoder.

\section{Soft Decoding}

LDPC soft decoder was introduced in [16]. For detectors, Hochwald et al. in [17] described a method to efficiently calculate the approximate LLRs from a list of candidates. It became possible to implement a soft output detector using (11).

$$
\begin{aligned}
& L_{E}\left(x_{k} \mid Y\right) \approx \frac{1}{2} x \in X_{k,+1}\left\{-\frac{1}{\sigma^{2}}\|y-H . s\|^{2}\right\}- \\
& \frac{1}{2} x \in X_{k,-1}\left\{\frac{1}{\sigma^{2}}\|y-H . s\|^{2}\right\}
\end{aligned}
$$

From the perspective of hardware design, the computation of LLR can be done in a separate unit. It keeps track of two numbers for each LLR; one for those whose $\mathrm{k}^{\text {th }}$ bit of the candidate list is 1 (Lambda-ML) and the other for 0 (Lambda-ML-bar). After that, the LLR values will be calculated as the subtraction of Lambda-ML and Lambda-ML-bar divided by two.

\section{LDPC Decoder}

An LDPC code is defined with parity check matrix called $\mathrm{H}$. Each row and column of the matrix is associated with parity check equation and received bits respectively. Parity check equation using Tanner graph is also called check nodes and the coded bits can be represented by variable nodes. A variable node is connected to a check node when the associated bit in $\mathrm{H}$ matrix is 1 . The process of decoding can be done with passing information through the edges of the graph. In this paper, we work with LDPC decoder based on our previous work presented in [18]-[19].

\section{RESULTS}

This section demonstrates the performance of our proposed soft decision based MIMO detector. The signal to noise ratio (SNR) is defined as the ratio of received information bit energy to noise variance.

As the case study we considered the IEEE 802.16e standard. This standard supports up to $4 \times 4$ antenna arrangement and modulation scheme of QPSK, 16QAM, and 64QAM. For performance evaluation, we simulated and demonstrated the BER versus SNR curves with different list sizes. All the simulation achieved with simulation of $10^{5}$ packet or at least presence of 100 error which happens first. To certain limit, the performance of both soft and hard detector will increase with the increase in list size and it will remain same for bigger list sizes. We first specified the optimum list size for each configuration (antenna arrangement and modulation scheme). Fig. 1 shows the BER versus SNR curve for hard decision after LDPC decoding for different modulation schemes. In the curves LDPC coded soft decoding, and LDPC coded hard decoding are represented by LCSD and LCHD respectively.

For hard decision LDPC decoder with QPSK modulation scheme, we can achieve maximum performance using list size of six. As demonstrated in Fig. 1 there is no improvement in the performance for list size of eight. In contrast if we decrease the value of $\mathrm{K}$ to 5 , then the performance loss is considerable. The minimum list size required to obtain maximum performance is 10 for 16QAM and 25 for 64QAM. The curves in the Fig. 1 are demonstrating the optimum derived list size in compare to the smaller and bigger list sizes. If we consider the list sizes higher than the mentioned ones, the performance does not improve while for smaller list sizes the loss in performance is considerable. Same analysis has been applied to derive the optimum list size for Soft decision decoding as the result is demonstrated in Fig. 2.

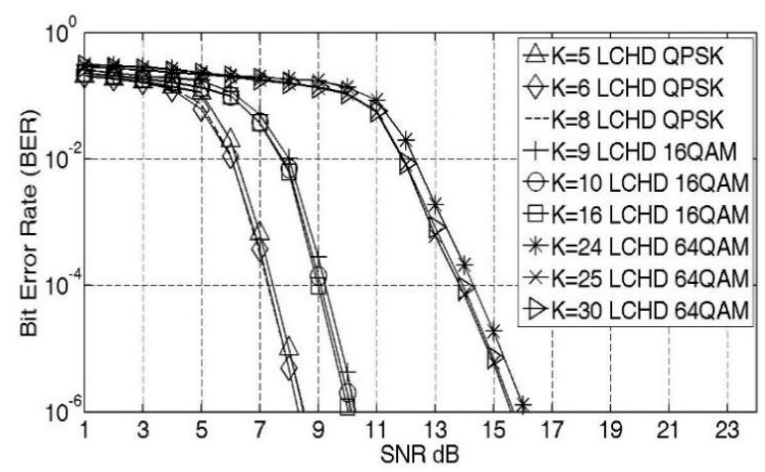

Fig. 1. BER versus SNR (Eb/No) curve of hard decision LDPC decoder.

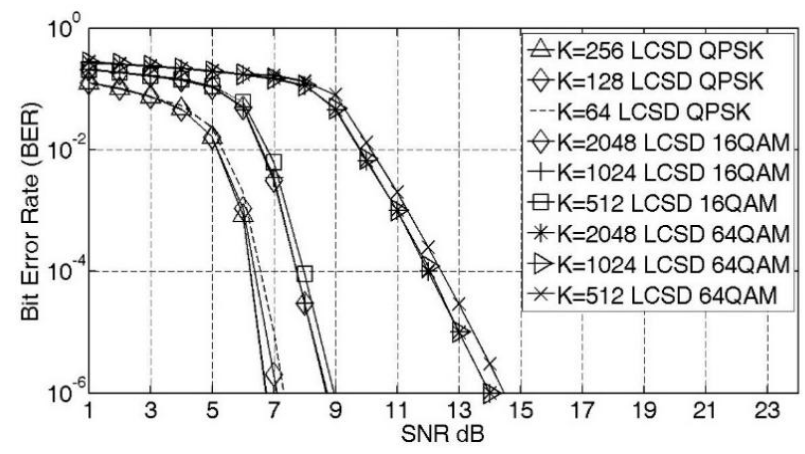

Fig. 2. BER versus SNR (Eb/No) curve of soft decision LDPC decoder.

For soft decision LDPC decoder, the optimum values of $\mathrm{K}$ are 256, 1024, and 1024 for QPSK, 16QAM, and 64QAM respectively. For soft decoding, we need bigger list than hard decoding. It is due to the fact that equation (11) ideally needs all the distances of tree to calculate the LLRs. It can be seen in Fig. 2 that, as long as the list size is large enough and the candidates of the list are those with the smallest distances (a "good enough" subset) a subset of candidates can be used to calculate the LLRs and there will be no performance loss. Conversely in hard decision decoding, we only consider the most probable candidate as our transmitted symbol. Therefore, the list size required for soft decision is dramatically higher than that of a hard one for a particular modulation scheme. The comparison of performance between hard and soft decoding for different modulation schemes is represented in Fig. 3.

As it is demonstrated in Fig. 3 a $1.5 \mathrm{~dB}$ improvement in performance can be achieved using LDPC coded soft decoding for QPSK modulation (at BER of $10^{-6}$ ). In addition, performance improvement between hard and soft decoding is $1.3 \mathrm{~dB}$ for both 16 and 64QAM at the same level of BER. Hence using soft decoding more than $1 \mathrm{~dB}$ improvement in performance can be achieved. 


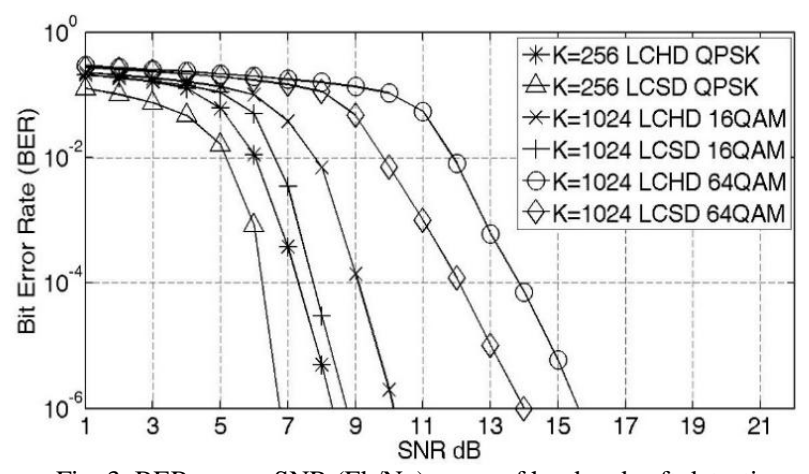

Fig. 3. BER versus SNR (Eb/No) curve of hard and soft detection.

The proposed scheme can be further improved by introducing LLR clipping [13]. By empirical analysis, we establish that limiting the LLR values can decrease the optimum list size. Fig. 2 shows that with unbounded LLR values, a list size of 256 is required for optimum performance in QPSK system. Both for 16QAM and 64 QAM, the required list size is 1024 . However, the same performance can be achieved with smaller list size by constraining the LLR values to a certain limit. Fig. 4-Fig. 6 show the performance for different values of K and LLR clipping.

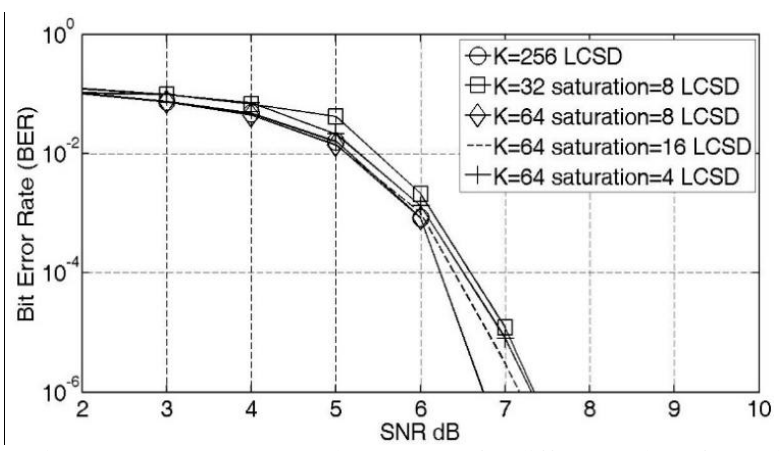

Fig. 4. BER versus SNR (Eb/No) curve for different value of $\mathrm{K}$ and saturation limit for QPSK MIMO system.

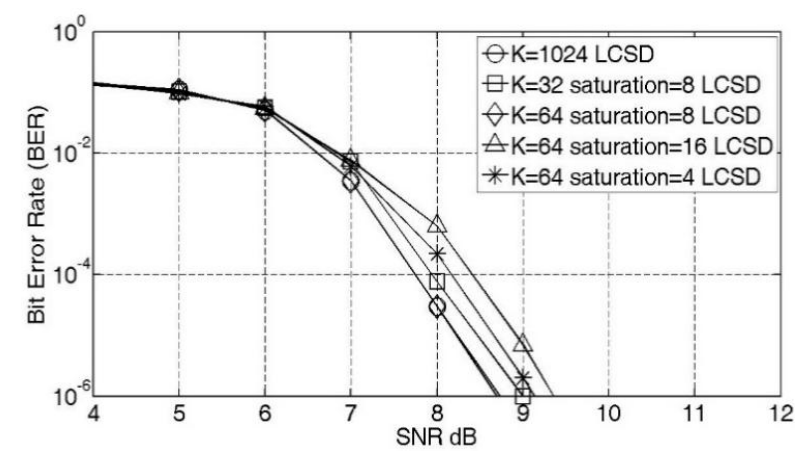

Fig. 5. BER versus SNR (Eb/No) curve for different value of $\mathrm{K}$ and saturation limit for 16QAM MIMO system.

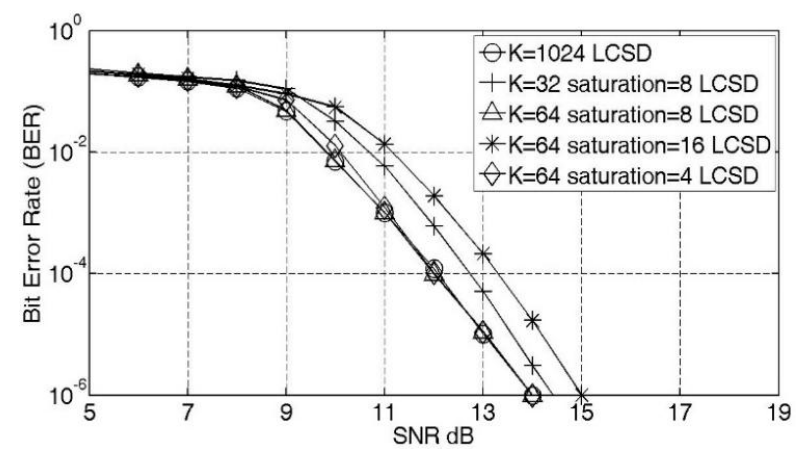

Fig. 6. BER versus SNR (Eb/No) curve for different value of $\mathrm{K}$ and saturation limit for 64QAM MIMO system.
It is evident from Fig. 4 that we can attain the optimum performance by keeping the list size equal to 64 and setting a saturation limit of eight, i.e. LLR values can change in $[-8,8]$ range. We have also included the curves for saturation limit of 4 and 16 with list size of 64 and also list size of 32 with saturation limit of eight to show the optimality of list size 64 and saturation limit of eight. All the curves show degraded or equal performance compared to list size of 64 and saturation limit of eight. All the parameters that provide equal performance have higher complexity (bigger list size or higher saturation limit), which is the reason that they have not been chosen as the optimum parameters. The optimum parameters can help us to achieve the same performance as the performance of very big unbounded list sizes. The same analogy can be applied for extracting the optimum list size and saturation limit for 16 QAM and 64 QAM. The performance curves for 16QAM and 64 QAM are presented in Fig. 5 and Fig. 6 respectively. The optimum parameters for both of the modulations are $\mathrm{K}=64$ and saturation limit of eight. From Fig 5, we can also observe that using the derived parameters same performance as of list size 1024 with unbounded LLR values in 16QAM modulation scheme can be reached. For 64QAM, we can use K as 64 and keep the saturation limit to either 4 or 8 to achieve the best performance achievable by our method. Thus the optimum values of list size and saturation limit are 64 and 4 respectively and the performance curves are shown in Fig. 6. Hence applying the saturation limit on the LLR values in LR-aided algorithm will result in $4 \mathrm{x}$ reduction in list size as well as in the complexity of detector and LLR calculation unit.

In [13] the effect of LLR clipping is investigated on the optimum list size required by K-best algorithm and it has been shown that it cannot reduce the list size. The combination of LR and MMSE extension improves the achievable performance and also changes the property of K-best algorithm. This results in smaller required list size to achieve maximum performance. In the next section we will conclude our results.

\section{CONCLUSION}

In this paper, we proposed a soft decision based MMSE extended K-best decoder exploiting the lattice reduction. Our improved MIMO soft detection algorithm has achieved 1.3 to $1.5 \mathrm{~dB}$ improvement comparing with hard detection. This paper also reports the optimized candidate list size and optimum saturation limit that should be applied for each modulation. We show that unlike the conventional K-best, LLR clipping can reduce the size of the optimum candidate list for new algorithm more than four times compared to conventional K-best and more than 8x compared to unclipped system with new algorithm.

\section{REFERENCES}

[1] IEEE Standard for Information Technology-LOCAL and Metropolitan Area Networks-Specific Requirements, Part 11: Wireless LAN Medium Access Control (MAC) and Physical Layer (PHY) Specifications Amendment 5: Enhancements for Higher Throughput, IEEE Standard 802.11n-2009, pp. 1-565, 2009.

[2] J. Jalden and B. Otterston, "On the complexity of sphere decoding in digital communications," IEEE Transaction on Signal Processing, vol. 53, no. 4, pp. 1474-1484, Apr. 2005. 
[3] C. Windpassinger and R. Fischer, "Low-complexity near-maximum likelihood detection and precoding for MIMO systems using lattice reduction," in Proc. IEEE Information Theory Workshop, Paris, France, Mar. 2003, pp. 345-348.

[4] X. Ma and W. Zhang, "Performance analysis for MIMO systems with lattice-reduction aided linear equalization," IEEE Transaction on Communication, vol. 56, no. 2, pp. 309-318, Feb. 2008

[5] Y. H. Gan, C. Ling, and W. H. Mow, "Complex lattice reduction algorithm for low-complexity full diversity MIMO detection," IEEE Transaction on Signal Processing, vol. 57, no. 7, pp. 2701-2710, Jul. 2009.

[6] B. M. Hochwald and S. ten Brink, "Achieving near-capacity on a multiple-antenna channel," IEEE Transactions on Communications, vol. 51, pp. 389-399, 2003.

[7] J. Ketonen, M. Juntti, and J. R. Cavallaro, "Performance-complexity comparison of receivers for a LTE MIMO-OFDM system," IEEE Transactions on Signal Processing, vol. 58, pp. 3360-3372, 2010.

[8] X. Qi and K. Hold, "A lattice-reduction-aided soft demapper for highrate coded MIMO-OFDM systems," IEEE Signal Processing Letter, vol. 15, no. 5, pp. 305-308, May 2007

[9] M. Shabany and P. G. Gulak, "The application of lattice-reduction to the K-Best algorithm for near-optimal MIMO detection," in Proc. IEEE International Symposium on Circuits and Systems (ISCAS), May 2008, pp. 316-319.

[10] C. P. Schnorr and M. Euchner, "Lattice basis reduction: Improved practical algorithms and solving subset sum problems," Mathematical Programming, vol. 66, pp. 181-191, 1994.

[11] Q. Zhou and X. Ma, "An Improved LR-aided k-best algorithm for MIMO detection," in Proc. IEEE International Conference on Wireless Communication and Signal Processing, 2012.

[12] Q. Wen, Q. Zhou, C. Zhao, and X. Ma, "Fixed-point realization of lattice-reduction aided MIMO receivers with complex k-best algorithm," in Proc. IEEE International Conference on Acoustics, Speech, and Signal Processing, 2013.

[13] M. Myllyla, J. Antikainen, M. Juntti, and J. R. Cavallaro, "The effect of LLR clipping to the complexity of list sphere detector algorithms," in Proc. the Forty-First Asilomar Conference on Signals, Systems and Computers, 2007, pp. 1559-1563.

[14] E. Agrell, T. Eirksson, A. Vardy, and K. Zeger, "Closest point search in lattices," IEEE Transaction on Information Theory, vol. 48, no. 8, pp. 2201-2214, Aug. 2002

[15] J. Jalden and P. Elia, "DMT optimality of LR-aided linear decoders for a general class of channels, lattice designs, and system models," IEEE Transaction on Information Theory, vol. 56, no. 10, pp. 4765-4780, Oct. 2010.

[16] J. Boutros, O. Pothier, and G. Zemor, "Generalized low density (Tanner) codes," in Proc. 1999 IEEE International Conference on Communications, 1999, vol. 441, pp. 441-445.

[17] C. Roth, P. Meinerzhagen, C. Studer, and A. Burg, "A 15.8 pJ/bit/iter quasi-cyclic LDPC decoder for IEEE 802.11n in $90 \mathrm{~nm}$ CMOS," Solid State Circuits Conference, 2010, pp. 1-4.

[18] K. Gunnam, G. Choi, W. Weihuang, and M. Yeary, "Multi-rate layered decoder architecture for block LDPC codes of the IEEE 802.11N wireless standard," in Proc. IEEE International Symposium on Circuits and Systems, 2007, pp. 1645-1648.

[19] K. K. Gunnam, "Area and energy efficient VLSI architectures for low density parity check decoders using an on-the-fly computation," Ph.D dissertation, Department of ECE, Texas A\&M University, College Station, TX, 2006

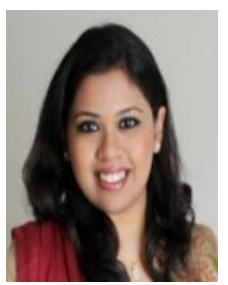

Mehnaz Rahman was born in Dhaka, Bangladesh on March 20, 1987. She completed her bachelor of science in electrical and electronics engineering from Bangladesh University of Engineering and Technology in 2011. She is currently doing $\mathrm{PhD}$ in computer engineering from Texas A\&M University, College Station, TX 77840 since Fall 2012 under the supervision of Dr. Gwan Choi. Her current research is focused on digital integrated circuits design on communication

After completion of her BS.c degree, she worked in Samsung R\&D Center, Dhaka, Bangladesh in 2011. She also did her internship in Intel Research Lab, Hillsboro, Oregon for summer, 2013 and submitted a patent which is now under the consideration of Intel Legal Committee.

Mrs. Rahman had got the Intel Research Award during her last internship in summer, 2013

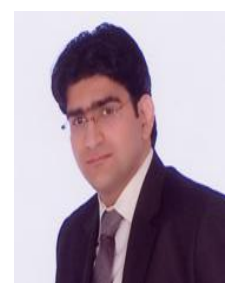

Ehsan Rohani was born in Tehran, Iran on June 2, 1983. He got his BS.c in electronic engineering from Amirkabir University (Tehran Polytechnic), Iran in 2003. He received his M.Sc. in electronic engineering, from ECE dept., University of Tehran, Iran in 2006. His research was on the simulation, fix point extraction, and implementation of base band of IEEE standards (IEEE 802.16). He has been working toward his $\mathrm{Ph}$. D since 2010 and as a Ph. D. candidate at Texas A\&M University, College Station, Texas, USA, where he is also a teaching assistant. He is also a research assistant in VLSI Signal Processing Lab, computer engineering group.

He tought (in 2006-2010) math, robotic, and electronic in Tehran schools and has teaching experience in different universities as a lecturer. He is a contributor to the VLSI, telecomunication, and signal processing society since 2005 where has poblished numerous journal and conference papers.

Mr. Rohani is currently working on low power physical layer implementation of MIMO system. His research interests lies in the area of baseband modeling and implementation of digital communication systems, low power techniques for VLSI circuits and DSPs, fixed point model extraction of digital circuits, digital signal processing, computer arithmetic, hardware and software implementation, system simulation, digital filter design, circuit level design of digital circuits, hardware simulation and implementation of digital systems.

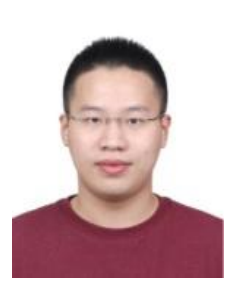

Jingwei Xu received the B.S. degree from Department of Electronic Engineering, Tsinghua University, Beijing, China, in 2011, and is currently working toward the Ph.D. degree at the Texas A\&M University.

His current research is focused on digital signal processing and digital integrated circuits design on communication and compressive sensing.

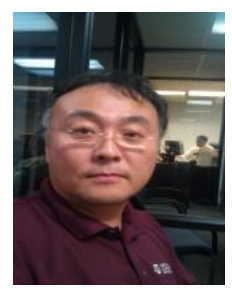

Gwan S. Choi received the B.S., M.S., and Ph.D degrees all in electrical and computer engineering from University of Illinois, Urbana-Champaign, IL, in 1989, 1990, and 1994 respectively. He has been a faculty member at the Department of Electrical and Computer Engineering, Texas A\&M University, College Station, since 1994.

His research interests include VLSI design for reliability and performance, low-power communication ASICs, multimedia processors, radiation detection sensor, and process-variation-tolerance. He has authored over one hundred peer-reviewed articles in the area. He has worked for Cray Research Inc. in 1987, Tandem Computers in 1990, and was a visiting scientist at NASA Langley Research Center in 1991. He has won research gifts and grants from National Science Foundation, NASA, IBM, AT\&T, LSI-Logics, Texas Higher-Education Coordinating Board and Department of Homeland Security. He has supervised $\mathrm{Ph} . \mathrm{D}$. research for nine students. He has won NSF CAREER award and has served as vice-chair of IEEE International Performance and Dependability Symposium, IPDS2000 and as committee member for a number of conferences and workshops. 